\title{
Resolución numérica de un modelo físico que evalúa la variación temporal de la concentración de radionucleidos vertidos en un río \\ Fabio Oscar López
}

\section{Resumen}

En este trabajo se presenta la resolución numérica de un modelo físico sencillo que permite evaluar la variación temporal de la concentración de actividad de radionucleidos vertidos en aguas de un río caudaloso.

El modelo físico resuelto, es un modelo sencillo del tipo mezcla completa similar al propuesto por el Organismo Internacional de Energía Atómica (OIEA) en la publicación SRS No 19 - "Generic Models for Use in Assessing the Impact of Discharges of Radioactive Substances to the Environment".

El modelo planteado es resuelto numéricamente mediante un método de diferencias finitas explícito.

Esta metodología puede ser una herramienta muy útil para estimar los niveles de la concentración de actividad de radionucleidos en aguas del río en el cual una instalación nuclear vierte sus efluentes líquidos.

Palabras clave: Dispersión de radionucleidos. Modelos físicos de mezcla completa. Resolución numérica. 


\section{Abstract}

This paper presents the numerical resolution of a physical model that allows to evaluate the temporal variation of the activity concentration of radionuclide discharges into waters of a river. The determined physical model is a simple model of complete mixing type similar to that proposed by the IAEA in the publication Safety Reports Series No. 19 - "Generic Models for Use in Assessing the Impact of Discharges of Radioactive Substances to the Environment".

The proposed model is solved numerically using an explicit finite difference method. This methodology can be a useful tool for estimating levels of activity concentration of radionuclides in river waters in which the nuclear installation discharges its liquid effluents.

Key words: Radionuclides dispersion. Physical model of complete mixing type. Numerical resolution. 


\section{Introducción}

La hidrosfera constituye una vía importante por la cual los materiales radiactivos emitidos por una instalación nuclear pueden dispersarse en el medio ambiente y por lo tanto llegar al hombre. Es por ello fundamental contar con herramientas que permitan predecir, con cierto grado de precisión, las variaciones temporales de la concentración de un contaminante vertido por una instalación.

En este trabajo se estudia la variación temporal de la concentración de actividad de radionucleidos en aguas de un río caudaloso, debida a una descarga puntual de estos radionucleidos efectuada por una central nuclear emplazada a orillas del curso de agua. Para llevar a cabo este estudio se plantea un modelo físico sencillo que es resuelto numéricamente mediante un método de diferencias finitas. Los resultados obtenidos pueden ser utilizados para analizar la evolución temporal de la concentración de actividad de los radionucleidos en aguas del río, en aquellos casos que se efectúen descargas puntuales programadas o descargas que se produzcan en forma accidental.

El modelo físico que se aplica es el propuesto por el OIEA en el Safety Reports Series No 19 (IAEA, 2001). En este modelo se considera que el material es descargado sobre una de las márgenes de un canal rectilíneo, en el cual los parámetros geométricos y las características del flujo (caudal, velocidad del flujo, dirección del mismo) no se modifican en forma significativa.

El modelo es aplicable a un río sin influencia de mareas, en el cual puede asumirse mezcla completa del contaminante. Es decir, puede asumirse que la concentración del contaminante estudiado es aproximadamente constante en cada sección transversal (no varía significativamente a lo largo de una línea perpendicular a las márgenes).

\section{Desarrollo}

\subsection{Caracterización general de los ríos}

A continuación se presentan las ecuaciones básicas que permiten estudiar el transporte de contaminantes en ríos, las cuales, son utilizadas para resolver problemas reales apelando a simplificaciones adecuadas (MENÉNDEZ, A. ,2010; PRARIO, B.E. et al., 2012).

$\frac{\partial C w_{, t o t}}{\partial t}+U \frac{\partial C w_{, t o t}}{\partial x}+V \frac{\partial C w_{, t o t}}{\partial y}+W \frac{\partial C w_{, t o t}}{\partial z}=\varepsilon_{x} \frac{\partial^{2} C w_{, t o t}}{\partial x^{2}}+\varepsilon_{y} \frac{\partial^{2} C w_{, t o t}}{\partial y^{2}}+\varepsilon_{z} \frac{\partial^{2} C w_{, t o t}}{\partial z^{2}}-\lambda_{i} C w_{, t o t}+S$

\begin{tabular}{|c|c|c|c|c|}
\hline $\begin{array}{c}\text { Variación } \\
\text { temporal }\end{array}$ & Transporte Advectivo & Transporte Difusivo & $\begin{array}{c}\text { Decaimiento } \\
\text { radiactivo }\end{array}$ & $\begin{array}{c}\text { Fuentes } \\
\text { externas }\end{array}$ \\
\hline
\end{tabular}


Donde:

$C w$, tot es la concentración del radionucleido $(\mathrm{Bq} / \mathrm{m} 3)$;

$U, V, W$ son las velocidades en las direcciones $\mathrm{x}, \mathrm{y} \mathrm{y} \mathrm{z}$, respectivamente $(\mathrm{m} / \mathrm{s})$;

$S$ es la fuente de adición o extracción del radionucleido $\left(\mathrm{Bq} / \mathrm{m}^{3} \cdot \mathrm{s}\right)$;

$t$ es el tiempo (s);

$x, y, z$ son las direcciones longitudinal, transversal y vertical, respectivamente, en coordenadas cartesianas.(m);

$\varepsilon x, \varepsilon y, \varepsilon z$ son los coeficientes de dispersión en las direcciones $x, y$ y $z$ respectivamente $\left(\mathrm{m}^{2} / \mathrm{s}\right)$;

$\lambda i$ es la constante de decaimiento del radionucleido $\left(\mathrm{s}^{-1}\right)$.

El transporte advectivo, representado por los últimos tres términos del primer miembro de la ecuación precedente, representa la entrada y salida de sustancia desde el volumen de control con la velocidad de la corriente.

Por su parte, el transporte difusivo, representado por los tres primeros términos del segundo miembro de la ecuación, se refiere a la entrada y salida de sustancia debido al efecto combinado de las difusiones molecular y turbulenta. Ambos efectos difusivos son causados por movimientos pulsátiles:

- El movimiento browniano (inducido térmicamente) de la molécula.

- Las fluctuaciones de las velocidades de la corriente alrededor de sus valores medios causadas por la turbulencia.

Usualmente, y tal como se expresa en la ecuación, ambos fenómenos son descriptos por la Ley de Fick, que establece que el transporte de masa en la dirección del gradiente de concentración es proporcional a ese gradiente a través de un coeficiente de difusión.

\subsubsection{Modelo Unidimensional}

Cuando la escala espacial de análisis de la hidrodinámica de un río es mayor que su ancho y su profundidad, es suficiente y conveniente plantear las ecuaciones de movimiento integradas en toda la sección transversal. Esto conduce a un modelo matemático unidimensional, en el cual los efectos de las escalas transversales de movimiento aparecen promediados y sólo se resuelven las escalas longitudinales largas.

La concentración del radionucleido vertido sobre el curso de agua en cualquier punto $\mathrm{x}$, puede representarse por su valor promedio $\mathrm{C}$ sobre toda la sección transversal. Si además se considera que no existen intercambios con el medio, la ecuación del modelo planteado es: 


$$
\begin{gathered}
\frac{\partial C}{\partial t}+U \frac{\partial C}{\partial x}=\varepsilon_{x} \frac{\partial^{2} C}{\partial x^{2}}-\lambda_{i} C+S \\
\text { siendo } \mathrm{C}\left(\mathrm{t}_{0}\right)=\mathrm{C}_{0} \quad \text { en el rango } \mathrm{t}_{0}<\mathrm{t}<\mathrm{t}_{\mathrm{f}}
\end{gathered}
$$

Donde:

$C$ es la concentración del radionucleido $(\mathrm{Bq} / \mathrm{m} 3)$;

$U$ es la velocidad en la dirección $x(\mathrm{~m} / \mathrm{s})$;

$S$ es la fuente de adición o extracción del radionucleido $\left(\mathrm{Bq} / \mathrm{m}^{3} \bullet \mathrm{s}\right)$;

$t$ es el tiempo (s);

$x$, es la dirección longitudinal. (m);

$\varepsilon x$, es el coeficiente de dispersión en la dirección $\mathrm{x}\left(\mathrm{m}^{2} / \mathrm{s}\right)$;

$\lambda i$ es la constante de decaimiento del radionucleido $\left(\mathrm{s}^{-1}\right)$.

Según lo expuesto en (IAEA, 2001), el coeficiente de dispersión en la dirección $x$ puede tomar la forma de la siguiente expresión:

$$
\varepsilon_{x}=\frac{U^{2} B^{2}}{f D u_{+}}
$$

Donde:

$B$ es el ancho de la sección del río (m)

$D$ es la profundidad promedio de la sección del cauce estudiado (m)

$u_{*}$ es la velocidad de corte $(\mathrm{m} / \mathrm{s})$

$f$ es un parámetro adimensional que depende de los parámetros hidrológicos del cauce.

La velocidad de corte y el parámetro adimensional $\mathrm{f}$ son definidos empíricamente y pueden tomar valores muy diferentes, dependiendo fundamentalmente de los parámetros hidrológicos de cada curso de agua bajo estudio (IAEA, 2001; MENÉNDEZ, A., 2010; PRARIO, B.E. et al, 2012; SAYRE, N.W. et al, 1973; LEOPOLD, L.B. et al, 1964; BOWIE, G.L. et al, 1985; MONTE, L. et al, 2009).

La ecuación (2) se resuelve en forma numérica a través de un método de diferencias finitas explícito.

\subsection{Método de diferencias finitas}

El método de diferencias finitas es una clásica aproximación utilizada para encontrar la solución numérica de las ecuaciones diferenciales que gobiernan el modelo matemático de un sistema continuo.

Este método consiste esencialmente en reemplazar la ecuación diferencial parcial que describe el problema por un número finito de ecuaciones algebraicas. Para esto 
las derivadas parciales se sustituyen por expresiones con los valores de la variable dependiente en un limitado número de puntos seleccionados.

A continuación se presenta la discretización propuesta en este estudio para resolver la ecuación planteada en (2). El método empleado es un método explícito centrado. Este es un método consistente desde el punto de vista numérico (BURDEN, R. L. et al, 1985; MARSHALL, G., 1985). En la figura 1 se muestra el esquema empleado para la discretización.

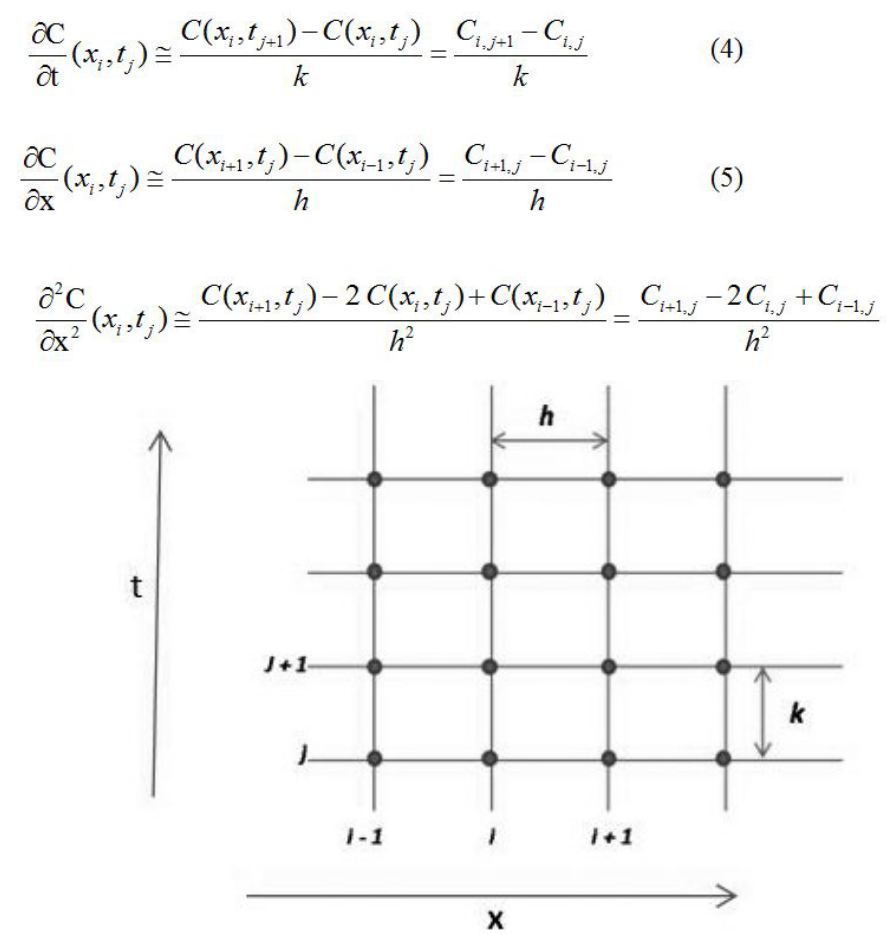

Figura 1: Esquema de la discretización empleada

Por lo tanto, si se reemplazan las derivadas parciales en (2), por las ecuaciones (4), (5) y (6) se concluye en la siguiente expresión:

$$
\frac{\mathrm{C}_{\mathrm{i}, j+1}-C_{i, j}}{\mathrm{k}}+U \frac{C_{i+1, j}-C_{i-1, j}}{h}=\varepsilon_{x}\left(\frac{C_{i+1, j}-2 C_{i, j}+C_{i-1, j}}{h^{2}}\right)-\lambda C_{i, j}+S
$$


A partir de esta ecuación se obtiene:

$$
\mathrm{C}_{\mathrm{i}, j+1}=C_{i, j}-k U \frac{C_{i+1, j}-C_{i-1, j}}{h}+k \varepsilon_{x}\left(\frac{C_{i+1, j}-2 C_{i, j}+C_{i-1, j}}{h^{2}}\right)-k \lambda C_{i, j}+k S
$$

Reagrupando convenientemente se llega a la siguiente expresión:

$$
\mathrm{C}_{\mathrm{i}, \mathrm{j}+1}=C_{i+1, j}(M-N)+C_{i, j}(1-2 M-k \lambda)+C_{i-1, j}(M+N)+k S
$$

Donde M y N son:

$$
M=\varepsilon_{x} \frac{k}{h^{2}} \quad, \quad N=U \frac{k}{2 h}
$$

\section{Resultados}

En esta sección, a modo de ejemplo de aplicación del modelo planteado, se presentan los resultados obtenidos en la resolución numérica del modelo físico unidimensional de transporte de contaminantes en un curso de agua, planteado en la ecuación (2), mediante el método de diferencias finitas explícito presentado en la sección 2.2 de este trabajo.

La resolución del modelo se realiza para el caso de una liberación puntual de tritio (H-3). El tritio es un radionucleido que se genera por la activación neutrónica del deuterio del agua pesada durante la operación normal de una central nuclear que utiliza uranio natural como combustible y agua pesada como refrigerante y moderador.

A continuación se presentan los parámetros del río y los parámetros de la discretización considerados, para resolver numéricamente el problema físico planteado:

\section{Parámetros del río:}

- Ancho de la sección del río - $\mathrm{B}=280 \mathrm{~m}$

- Profundidad del cauce - $\mathrm{D}=12,5 \mathrm{~m}$

- Sección transversal del canal propuesto en este estudio $=3500 \mathrm{~m}^{2}$.

- Caudal del río - $\mathrm{q}=4000 \mathrm{~m}^{3} / \mathrm{s}$

- Velocidad en la dirección $x-\mathrm{U}=1,1 \mathrm{~m} / \mathrm{s}$

- Velocidad de corte - $\mathrm{u}^{*}=0,1 * \mathrm{U}=0,11 \mathrm{~m} / \mathrm{s}^{1}$. (IAEA, 2001).

- Parámetro adimensional $-f=100$ 
Como fue expresado en la sección 2.1.1, el parámetro adimensional $f$ depende fundamentalmente de los parámetros hidrológicos del cauce bajo estudio, y su valor es determinado en forma empírica. El rango de valores presentado en la literatura para este parámetro adimensional es muy amplio. (MENÉNDEZ, A., 2010; PRARIO, B.E. et al, 2012; SAYRE, N.W. et al, 1973; LEOPOLD, L.B. et al, 1964; BOWIE, G.L. et al, 1985; MONTE, L. et al, 2009). El valor considerado en este trabajo se encuentra dentro del rango de los registros correspondientes a los ríos caudalosos.

\section{Parámetros de la discretización:}

- $\mathrm{k}=300 \mathrm{~s}$

$\cdot \mathrm{h}=1000 \mathrm{~m}$

Estos valores aseguran la estabilidad y la convergencia numérica de la resolución (BURDEN, R. L. et al, 1985; MARSHALL, G., 1985).

Para llevar a cabo la resolución numérica del modelo físico planteado se trabaja sobre una descarga unitaria que es liberada en un corto lapso de tiempo. De esta manera se intenta simular una descarga puntual de tritio realizada por la central.

Se postula una tasa de descarga $\left(\mathrm{Q}^{*}\right)$ unitaria de $1 \mathrm{~Bq}$ por segundo de tritio contenido en $1 \mathrm{~m} 3$ durante un período de tiempo de 5 minutos.

$$
\mathrm{Q}^{*}=1 \mathrm{~Bq} / \mathrm{m}^{3} \text { s. durante } \Delta \mathrm{t}=300 \mathrm{~s}
$$

Como condición inicial se considera que la concentración de tritio en el agua del río es nula, o sea

$$
\mathrm{C}\left(\mathrm{t}^{0}\right)=\mathrm{C}_{0}=0
$$

En las siguientes figuras se presenta la evolución temporal de la concentración de tritio en diferentes puntos de observación. En todos los casos se muestran los valores de concentración relativos al valor máximo obtenido en la primera etapa analizada.

En la figura 2 se observa la evolución en el tiempo de la concentración de actividad de tritio en un punto ubicado a $1000 \mathrm{~m}$ de la descarga. 


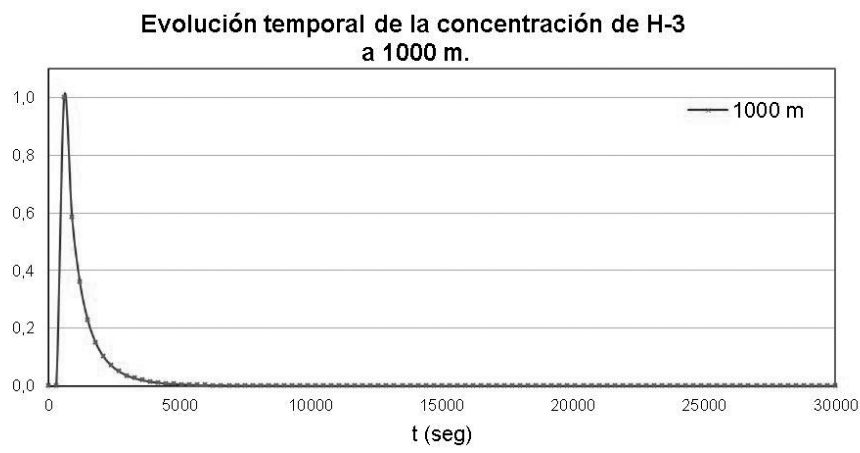

Figura 2. Evolución temporal de la concentración de tritio en aguas del río analizada a una distancia de $1000 \mathrm{~m}$ desde el punto de descarga.

En la figura 3 se presenta la evolución temporal en el rango de 1000 a 5000 metros. Se observa que las diferencias entre los valores máximos disminuyen a medida que se incrementa la distancia desde el punto de descarga.

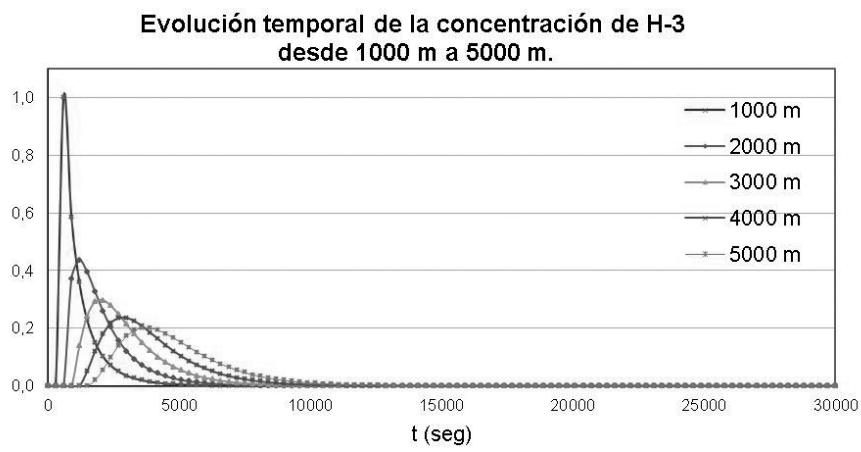

Figura 3. Evolución temporal de la concentración de tritio en aguas del río en el rango de 1000 a $5000 \mathrm{~m}$ desde la descarga.

En la figura 4 se presenta la evolución temporal en el rango de 1000 a 10000 metros. Se verifica que se cumple con la tendencia expuesta en los casos anteriores. Se observa que la diferencia entre los valores máximos de concentración disminuye punto a punto a medida que se incrementa la distancia desde el punto de descarga. 


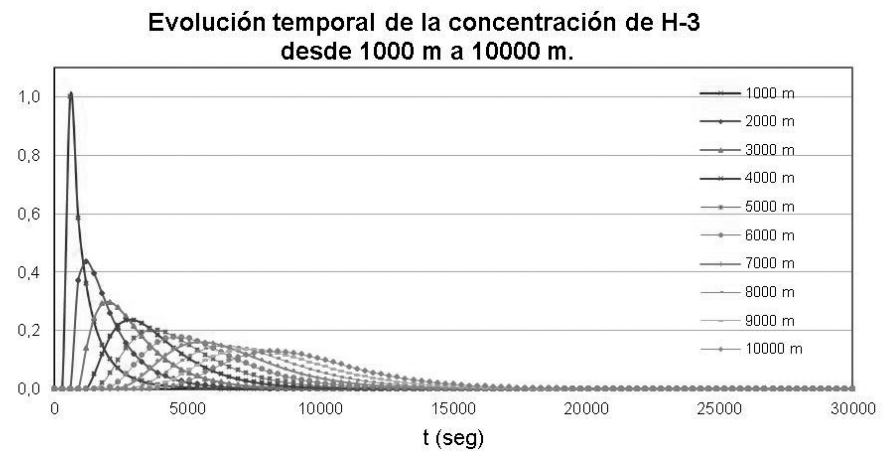

Figura 4. Evolución temporal de la concentración de tritio en aguas del río en el rango de 1000 a $10000 \mathrm{~m}$ desde la descarga.

En la siguiente figura se muestran las variaciones temporales de la concentración de tritio en los puntos ubicados a 5000, 10000, 15000 y 20000 metros desde el punto de la descarga. A partir de esto se puede observar que la herramienta desarrollada puede ser empleada en un amplio rango de distancias.

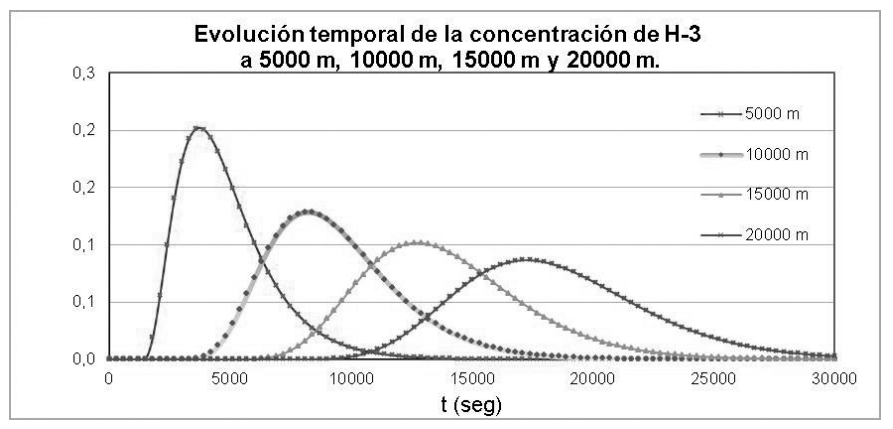

Figura 5. Evolución temporal de la concentración de tritio en aguas del Río a 5000, 10000, 15000 y $20000 \mathrm{~m}$ desde la descarga.

\subsection{Comparación de los valores máximos de concentración estimados en cada punto.}

En la siguiente tabla se presenta la relación porcentual entre cada valor máximo calculado con el inmediato anterior.

$$
p=\frac{C_{\max , i+1}}{C_{\max , i}} * 100
$$




\begin{tabular}{|c|c|c|c|}
\hline Distancia $(\mathbf{m})$ & Porcentaje $(\%)$ & Distancia $(\mathbf{m})$ & Porcentaje $(\%)$ \\
\hline 1000 & --- & 11000 & 94,5 \\
\hline 2000 & 43,6 & 12000 & 95,0 \\
\hline 3000 & 68,2 & 13000 & 95,4 \\
\hline 4000 & 79,4 & 14000 & 95,8 \\
\hline 5000 & 85,2 & 15000 & 96,1 \\
\hline 6000 & 88,4 & 16000 & 96,4 \\
\hline 7000 & 90,4 & 17000 & 96,6 \\
\hline 8000 & 91,9 & 18000 & 96,9 \\
\hline 9000 & 93,0 & 19000 & 97,0 \\
\hline 10000 & 93,8 & 20000 & 97,2 \\
\hline
\end{tabular}

Tabla 1. Relación porcentual respecto al valor anterior

En la siguiente figura se representa gráficamente esta variación porcentual.

Relación porcentual entre los valores máximos de concentración de $\mathrm{H}-3$.

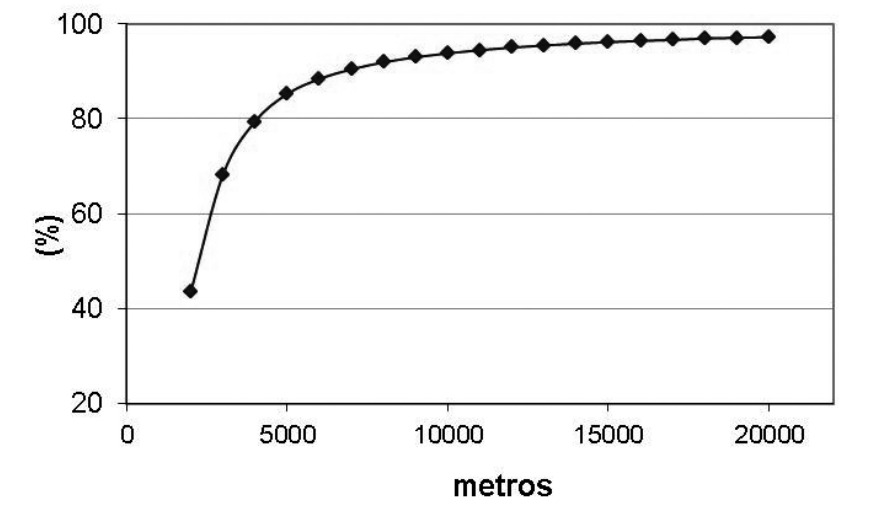

Figura 6. Relación porcentual entre los valores máximos de concentración de actividad de tritio

A partir de lo expuesto en esta figura se puede asegurar que los valores máximos de concentración de actividad del radionucleido descargado en aguas del río tienden a un valor constante. 


\section{Conclusiones}

En este trabajo se presenta una metodología para resolver en forma numérica un modelo físico sencillo que permite evaluar la evolución temporal de la concentración de actividad de radionucleidos en aguas de río, debido a una descarga puntual realizada por una central nuclear.

El modelo físico resuelto es un modelo unidimensional del tipo mezcla completa, la resolución del mismo se realiza a través de un método de diferencias finitas explícito, y se utilizan parámetros hidrológicos de la zona.

Sobre la base de los resultados obtenidos, para el ejemplo propuesto de descarga de tritio, se concluye que en los primeros tramos analizados es importante la disminución del valor máximo de concentración de tritio entre los puntos de observación contiguos, y luego esta diferencia decrece notoriamente. Después de una distancia de 12000 metros desde el punto de descarga las diferencias entre los puntos de observación adyacentes son inferiores al 5\%. A partir de esto se puede concluir que, después de una primera etapa, la concentración de actividad de tritio en aguas del río tiende a un valor constante.

Esta metodología puede ser una herramienta muy útil para estimar el orden de magnitud de los niveles de concentración actividad de radionucleidos, con posterioridad a una descarga realizada por la central nuclear, en el agua del río, en las zonas localizadas aguas abajo de la instalación. Para ser utilizada en forma adecuada, esta herramienta debe ser previamente validada. 


\section{Referencias}

IAEA - INTERNATIONAL ATOMIC ENERGY AGENCY (2001). Generic Models for Use in Assessing the Impact of Discharges of Radioactive Substances to the Environment. Safety Reports Series Nº19, Viena.

MENÉNDEZ, A. (2010). Transporte de Contaminantes en el Medio Acuático. Universidad Tecnológica Nacional. Maestría en Ingeniería Ambiental. 2010.

PRARIO, B.E., DRAGANI, W., MEDIAVILLA, D.G., D'ONOFRIO, E. (2012). Hydrodynamic numerical simulation at the mouths of the Parana and Uruguay rivers and the upper Rio de la Plata estuary: A realistic boundary condition. Applied Mathematical Modelling. Vol.35. 5265-5275.

SAYRE, N.W., Natural mixing processes in rivers, (1973). Environmental Impact on Rivers (River Mechanics II), H.W. Shen, Fort Collins, CO.

LEOPOLD, L.B., WOLMAN, M.G., MILLER, J.P., (1964). Fluvial Processes in Geomorphology, W.H. Froeman, San Francisco, CA.

BOWIE, G.L., et al., (1985). Rates, Constants, and Kinetics Formulations in Surface Water Quality Modeling, 2nd edn, prepared for the US Environmental Protection Agency by Tetra Tech, Lafayette, CA, and Humbolt State University, Arcata, CA.

MONTE, L., PERIAÑEZ, R., BOYER, P., SMITH, J. and BRITTAIN, J. (2009). The role of physical processes controlling the behaviour of radionuclide contaminants in the aquatic environment: a review of state-of-the-art modelling approaches. Journal of Environmental Radioactivity 100. 779-784.

BURDEN, R. L. ; FAIRES, J. D. (1985). Análisis Numérico. Grupo Editorial Iberoamérica, México,

MARSHALL, G., (1985). Solución Numérica de Ecuaciones Diferenciales, Tomo 1, Ecuaciones Diferenciales Ordinarias, Editorial Reverté. Argentina. 
